\title{
Le Griot Dans La Litterature Postcoloniale: Une Etude De Guelwaar De Sembene Ousmane
}

\author{
Ifeoma Mabel Onyemelukwe, Abubakar Dauda Adamu Et \\ Chukwunonso Hyacinth Muotoo \\ http://dx.doi./org/10.4314/ujah.v22i1.3
}

\section{Résumé}

Le griot dans la société traditionnelle africaine de l'ère précoloniale est un personnage complexe chargé d'une myriade de fonctions. Il est bien respecté et honoré. La présente étude se donne l'objectif d'examiner profondément la peinture du griot dans la littérature africaine postcoloniale utilisant Guelwaar de Sembène Ousmane comme texte de base tout en établissant ses fonctions et ses portraits. L'étude privilégie quelques théories critiques comme le postcolonialisme, les théories marxiste et féministe. De plus, l'examen s'effectue à la lumière de l'image du griot d'antan et des sept catégories de nouveaux griots postulés par Ifeoma Mabel Onyemelukwe. Nous découvrons deux types de griot dépeints par Ousmane dans Guelwaar : le griot personnage littéraire nommé Guelwaar et le griot écrivain contemporain africain, Sembène Ousmane lui-même. Ces deux griots entretiennent des rapports de similitude et de divergence avec le vrai griot. Mais le griot écrivain contemporain africain ressemble beaucoup plus au griot d'antan en dépit des points de divergence. Nous finissons par déceler dix-huit fonctions du griot et par-là arriver aux dix-huit portraits du griot dans Guelwaar dont le griot bibliothèque publique bien documentée et le griot détenteur de la littérature écrite africaine postcoloniale. Nous parvenons à la conclusion que la littérature postcoloniale, comme l'atteste Guelwaar de Sembène Ousmane, se caractérise par une revalorisation des valeurs authentiques, honorables et louables 
du griot de caste. Ceci est symbolisé par l'apparition du griot personnage littéraire dans certaines œuvres de la littérature écrite postcoloniale africaine comme Guelwaar et surtout la prééminence accordée aux griots écrivains contemporains africains tel Sembène Ousmane.

\section{Mots clefs : Griot, postcolonialisme, littérature postcoloniale, fonction, portrait, marxisme, féminisme, Guelwaar, Sembène Ousmane.}

\section{Abstract}

The griot, in the traditional African society is a complex personality charged with multiple functions. He is well respected and honoured. The objective of the present research is to make an in-depth study of the depiction of the griot in post-colonial literature using Guelwaar as study text while establishing his functions and portraits. Postcolonialism, Marxist and Feminist theories constitute the theoretical framework. Furthermore, the examination is carried out in the light of the image of the real griot and the seven categories of new griots promulgated by Ifeoma Mabel Onyemelukwe. It is found that Sembène Ousmane depicts two types of griots in Guelwaar, namely: the griot as literary character named Guelwaar and the griot as contemporary African writer, Sembène Ousmane, himself. These two griots have certain similarities and differences with the real griot. But the findings show that the griot as contemporary African writer resembles more the real griot, their points of disparity notwithstanding. Eighteen functions and eighteen portraits of the griot are established, among which are: the griot as well documented library and the griot as custodian of written postcolonial African Literature. A firm conclusion is reached, that postcolonial literature, as reflected in Sembène Ousmane's Guelwaar, is characterized by 
the revalorization of the real griot's authentic, honorable and praiseworthy values. This is symbolized in the projection of the griot as literary character in some literary works like Guelwaar and in particular, the preeminence given to griots as Contemporary African writers as typified by Sembène Ousmane.

Keywords: griot, postcolonialism, postcolonial literature, function, portrait, Marxism, feminism, Guelwaar, Sembène Ousmane

\section{Introduction}

Le griot représente la caste inferieure ou Gnegno dans le système des castes sénégalais à l'ère précoloniale. Les castes inferieures au Sénégal comportent les ethnies vaincues qui s'occupent des œuvres spécialisées, par exemple, les Teugg ou les forgerons ou les orfèvres, les Oude ou les cordonniers, les Laobe ou les bûcherons ou les menuisiers. Leur nom collectif c'est les Dieuf-Lekk. A part les Dieuf-Lekk, il y a aussi les Sab-Lekk, les Baw-Lekket les Gnole.... Les Baw-lekk sont les griots proprement dits ou les guewal en wolof (Diop 50-52).

Ce propos de Mahjémout Diop dévoile assez clairement qu'au Sénégal, les griots, en général, appartiennent aux castes inferieures et qu'on peut faire des distinctions entre les griots - ceux qui 
s'occupent des œuvres spécialisées comme les forgerons et les cordonniers et certains autres dont les Baw-lekk. Ceux-ci sont les griots à proprement parler ; ils s'appellent les guewal en wolof. Chez les Maninke et Bambara, d'après Mamadou Kouyaté, l'équivalent de guelwal est jali ou jeli alors que les Peuls font usage du terme gewel ou gawla (221-222). Sembène Ousmane, le romancier, essayiste et cinéaste de grande renommée mondiale, qui fait l'objet de la présente étude, projette dans Véhi-Ciosane, le griot cordonnier et le griot ressemblant au vrai griot ouguelwar dans Guelwaar. Notons que Véhi-Ciosane est l'avant-coureur des romans postcoloniaux de cette icône de la littérature africaine. Alors, dans l'un et l'autre, Ousmane fait une peinture du griot à l'ère postcoloniale.

La description que nous livre Diop ci-dessus porte sur le griot pendant la période précoloniale. Un questionnement probant qui se lève ici porte sur l'évolution du griot dans la société africaine d'après les indépendances selon la peinture de Sembène Ousmane. Peut-on identifier un certain changement dans le cadre du métier et donc du griot dans la littérature postcoloniale ? Est-ce que le griot dans l'ère postcoloniale assume de nouvelles fonctions ? Peut-on établir de nouvelles espèces de griot dans la littérature postcoloniale en s'appuyant sur Guelwaar?

La présente étude a pour objectifs d'examiner la peinture du griot dans la littérature postcoloniale, d'établir ses fonctions, ses portraits et des écarts dans le métier du griot d'antan ; ceci en nous appuyant sur Guelwaar de Sembène Ousmane à part quelques intertextes. Pour effectuer cette recherche, nous utilisons trois théories critiques : le postcolonialisme, le marxisme et le féminisme. Le postulat d'Ifeoma Mabel Onyemelukwe concernant l'effritement du personnage du vrai griot et la prolifération de nouveaux griots dont elle a relevée sept catégories s'avère utile pour cette étude. 
Le point de démarrage commode est la définition et l'explication de certains mots clés : le postcolonialisme et la littérature postcoloniale. Ce qui suit, c'est la peinture du griot dans Guelwaar. Puis nous tâchons d'établir ses fonctions et portraits avant de glisser une conclusion.

\section{Explication de certains mots clés Le postcolonialisme}

Le postcolonialisme, aussi appelé le post-colonialisme, est une théorie critique qui se concerne avec l'étude des effets de colonisation sur les cultures et les sociétés. Selon que des historiens l'ont utilisé après la deuxième guerre mondiale dans les termes comme «Etats postcoloniaux », le mot «postcolonial » avait une signification plus ou moins chronologique ; il veut dire la période postindépendance. Oxford English Dictionary le définit comme ce qui a lieu après la colonisation (Onyemelukwe, Colonial 189). Pourtant, vers la fin des années 1970, les critiques littéraires tels que Spivak et Bhabha emploient le terme en contexte d'étude de divers effets culturels occasionnés par la colonisation (Ashcroft Bill, Gareth Griffiths et Helen Tiffin, Key Concepts 186). Remarquons d'emblée que le postcolonialisme a été fondé sur les principes de l'ouvrage d'Edouard Said intitulé Orientalism publié en 1978 (Onyemelukwe, Violence \&Politics 6). De plus, le terme « postcolonial » a été utilisé pour la première fois pour référer aux interactions culturelles au milieu des sociétés coloniales dans les cercles littéraires. (Ashcroft et al. 1977 cité par Ashcroft, Grifith\& Tiffin, Key Concepts 186). C'était en raison du jeu politique et la tentative de focaliser sur les intérêts des domaines tels que la littérature de Commonwealth et l'étude de nouvelles littératures d'expression anglaise, par exemple, la littérature africaine d'expression anglaise, qui ont vu le jour vers la fin de 1960. Le sens 
du terme « postcolonial » a évolué au point de désigner actuellement les expériences politiques, linguistiques et culturelles des sociétés des anciennes colonies européennes (Ashcroft, Grifiths\& Tiffin, Key Concepts 186 ; Ashcroft, Grifiths\& Tiffin, The Empire 187 ; Onyemelukwe, Violence and Politics 6).

De nos jours, l'on utilise le postcolonialisme ou le postcolonialisme dans une grande diversité de voies pour inclure l'étude et l'analyse des territoires conquis par les Européens, les diverses institutions issues de la colonisation européenne, les opérations discursives de l'empire, les péripéties de la construction du sujet dans le discours colonial et la résistance de ces sujets et le plus important, peut-être, les différentes réponses à ces incursions et leurs légataires coloniales contemporaines dans des nations et communautés pré- et postindépendances. Bien que le terme se penche à focaliser sur les productions culturelles de telles sociétés, on constate qu'il s'emploie largement dans les analyses historiques, politiques, sociologiques et économiques tant que ces disciplines continuent de s'intéresser aux effets de l'impérialisme européen sur les sociétés du monde (Ashcroft, Griffith \& Tiffin, Key Concepts 187). Donc, nous trouvons la théorie du post-colonialisme très apte pour le genre d'analyse commandé par la présente étude.

\section{La littérature postcoloniale}

Ainsi que l'a expliqué Ifeoma Onyemelukwe, la littérature postcoloniale décrit les expériences politiques, linguistiques et culturelles des sociétés des nations ou communautés colonisés préalablement par les impérialistes européens. Elle fait mention de communautés impliquées dans cette notion : les Indes, les Caraïbes, l'Amérique du sud et les pays africains (Violence \&Politics 6). Mishra et Hodge maintiennent que la littérature postcoloniale se caractérise par le syncrétisme et l'hybridité (281). 


\section{La peinture du griot dans Guelwaar : Un survol}

Rappelons bel et bien que pour effectuer la présente étude, nous nous appuyons sur le postcolonialisme, le marxisme, le féminisme ainsi qu'au postulat d'Onyemelukwe dont l'image du vrai griot et sept catégories de nouveaux griots, à savoir :

- Le griot musicien moderne

- Le griot politicien

- Le griot chanteur de louanges ambulant et parasitaire

- Le griot faux mendiant

- Le griot comédien moderne

- Le griot personnage littéraire

- Le griot écrivain contemporain africain (53-63).

Rappelons aussi que le vrai griot ou le griot d'antan tel qu'il se retrouvait dans la société traditionnelle africaine pendant l'ère précoloniale était « un personnage complexe, chargé de multiples fonctions, tenant plusieurs positions nobles qui lui confèrent le statut spécial d'un homme digne, honorable et vénérable jouissant d'une haute estime dans ladite société » (Onyemelukwe, La littérature orale 49). De plus, on est né griot, on ne le devient pas. Le métier de ce dépositaire de la littérature orale africaine était héréditaire, transmis de père en fils, de génération en génération. Cependant, dans le temps, le métier du griot a subi des mutations inévitables menant à l'effritement du personnage du griot d'antan et l'émergence de nouvelles catégories de griot qu'on vient de soulever. Nous convenons avec Onyemelukwe que ces mutations sont attribuables aux nombreux facteurs tels que la colonisation, l'islamisation, l'européanisation (l'école européenne), le modernisme (le développement technologique), les catastrophes environnementales (sècheresse, famine), la crise économique et 
l'emploi de la musique dans le sérail politique (La littérature orale 53). Un ajout important concernant les facteurs déclencheurs des changements incontournables qu'a subi le métier du griot d'antan ce sont les caractéristiques de syncrétisme et d'hybridité que manifeste la littérature postcoloniale (Mishra et Hodge 281). Tendance à l'originalité se perd dans la littérature postcoloniale face au syncrétisme et à l'hybridité ou au métissage moderne. On ne peut pas nier la possibilité de l'existence, de nos jours, des vestiges de vrais griots dans certaines parties d'Afrique.

Néanmoins, Guelwaar, d'après la peinture de Sembène Ousmane, ne présente pas l'occasion à l'auteur de dépeindre carrément la figure du vrai griot ou du griot authentique, cette figure dominante de la littérature orale africaine pendant la période précoloniale. Notre étude aboutit à la constatation que le célèbre romancier et cinéaste sénégalais Sembène Ousmane, dans son troisième roman postcolonial mais neuvième roman Guelwaar, publié en 1996, fait une peinture excellente de deux catégories de griot à en juger par le postulat d'Onyemelukwe. Ce sont : le griot personnage littéraire et le griot écrivain contemporain africain. Signalons d'emblée que ces deux catégories de griot entretiennent, quand même, des rapports de similitude et de divergence avec le griot d'antan. Ce fait sautera davantage aux yeux lors de notre examen des fonctions et portraits de ces deux types de griot.

Sembène Ousmane, un marxiste féministe, peint d'abord le griot personnage littéraire qui n'est d'autre personne que Pierre Henri Thioune, le prénommé Guelwaar, le héros de Guelwaar, un roman de désenchantement qui décrit vivement le bouleversement déclenché par l'ensevelissement d'un chrétien, Guelwaar lui-même, dans un cimetière musulman, un quiproquo occasionné par une faute administrative. Ce roman postcolonial aborde, en plus, la thématique de la sècheresse prolongée déclenchant la violence 
structurale (faim, disette, pauvreté, misère, mendicité etc.) et l'intervention de l'administration postcoloniale par son acceptation du don caritatif. Ce fait remonte à la mendicité nationale voire internationale. Notons que c'est sa deuxième fois de créer un tel griot dans son imaginaire littéraire à l'ère postindépendances. Il a, préalablement, projeté le personnage de DéthyèLaw dans VéhiCiosane ce qu'Onyemelukwe décrit comme «le précurseur du nouveau roman africain » (La littérature orale 61). Elle a classifié Déthyè Law comme l'antihéros type I (Heroism and Antiheroism 41, 47-48) dans le monde mimétique caractérisé par la lutte des classes, notamment : les nobles ou guellewar (les oppresseurs) visà-vis des serfs ou Gnégno(les opprimés); ce dernier représenté par le protagoniste symbolique, Déthyè Law. Celui-ci figure dans VéhiCiosane comme un des personnages de la fiction, un personnage littéraire d'où l'appellation appropriée le griot personnage littéraire. Il nous renvoie à la griotte dans Une si longue lettre de Mariama Bâ qui, elle aussi passera pour un griot personnage littéraire. Nous constatons qu'à la différence de Déthyè Law, qui est un griot cordonnier, Pierre Henri Thioune est un guelwar reflétant, en quelque sorte, le vrai griot comme son nom l'indique.

A part cette création littéraire, Sembène Ousmane se fait luimême un griot dans Guelwaar d'où sa catégorisation par Onyemelukwe comme le griot écrivain contemporain africain qu'on a déjà soulevé. Ainsi nous fait-il penser à un bon nombre de griots écrivains contemporains africains morts ou vivants tels que Chinua Achebe, Ahmadou Kourouma, Mongo Beti, Djibril Tamsir Niane, Mariama Bâ, Aminata Sow Fall, Alain Mabanckou, Wole Soyinka, Fatou Diome, Chimamanda Ngozi Adichie, Tierno Monénembo, Akachi Ezeigbo et Ifeoma Onyemelukwe. 


\section{Fonctions du griot dans Guelwaar}

Tableau I ci-dessous nous livre la découverte de la présente étude que le griot, selon que l'auteur de Guelwaar l'ait dépeint, se charge de dix-huit fonctions. Nous discutons tout de suite ces fonctions.

\section{Tableau I : Fonctions du griot dans Guelwaar de Sembène} Ousmane

\begin{tabular}{|l|l|}
\hline $\mathrm{N}^{\mathrm{O}}$ & Fonction de/d' \\
\hline 1. & Porte-parole du peuple \\
\hline 2. & Educateur du peuple \\
\hline 3. & Libérateur du peuple \\
\hline 4. & $\begin{array}{l}\text { Critique des maux socioéconomique et politiques de la } \\
\text { société }\end{array}$ \\
\hline 5. & Ami loyal et fraternel et homme du peuple \\
\hline 6. & $\begin{array}{l}\text { Harangueur du discours violent et ennuyeux contre les } \\
\text { autorités postcoloniales }\end{array}$ \\
\hline 7. & Incitant d'une révolte collective du peuple \\
\hline 8. & Défenseur de l'idéologie marxiste \\
\hline 9. & Promoteur de l'émancipation féminine \\
\hline 10. & Déconstructeur des préceptes religieux \\
\hline 11. & Maître de la parole \\
\hline 12. & Diseur de la vérité \\
\hline 13. & Historien \\
\hline 14. & Psychologue \\
\hline 15. & Conseiller \\
\hline 16. & Eveilleur de la conscience sociopolitique chez les gens \\
\hline 17. & Bibliothèque publique bien documentée \\
\hline 18. & Détenteur de la littérature écrite africaine postcoloniale \\
\hline
\end{tabular}


Pierre Henri Thioune surnommé Guelwaar est un griotnarrateur dans Guelwaar. Sa figure littéraire, comme l'a signalé Onyemelukwe, dépasse les fonctions géographiques et culturelles (La littérature orale 61). Il va sans dire qu'il n'a pas foncièrement l'image du vrai griot de la société sénégalaise à l'ère précoloniale. A la différence du griot d'antan dépeint dans Soundjata ou l'épopée mandingue de Djibril TamsirNiane, Guelwaar ne se lie à aucun roi, il n'est pas le porte-parole du roi mais il est plutôt le porte-parole de son créateur l'écrivain Sembène Ousmane. De plus, il n'est ni gardien de la mémoire collective, ni généalogiste, ni maître de cérémonies, ni conseiller du roi, contrairement au griot d'antan dépeint dans Soundjata ou l'épopée mandingue. Tout cela fait preuve de l'effritement du rôle du griot de caste en Afrique contemporaine évoqué par Niane et démontré par Onyemelukwe ( $\mathrm{La}$ littérature orale 61 ).

Guelwaar est marxiste et féministe tout comme Sembène Ousmane. En tant que féministe, il permet aux femmes de l'Association féminine Morom-yi présidée par Dame Véronique de se réunir chez lui pour discuter les problèmes socio-économiques entrainés par la sècheresse, entre autres. Nous apprenons par la bouche de Guelwaar que ces femmes «parlent de la famine, de la sècheresse, des détournements, de l'enrichissement illicite, de mariage, de divorce... de la situation catastrophique du pays (Guelwaar 32). Ces féministes croient que le mal du Sénégal d'après les indépendances s'enracine dans la sècheresse et la famine entre autres. Nous convenons avec René Dumont qui attribue le mauvais départ qu'a eu l'Afrique à de tels problèmes surtout la misère (Dumont 10-11). Guelwaar défend et protège ces femmes entre les mains des agents de police. Il se montre donc par ce rôle le promoteur de l'émancipation féminine ainsi qu'un ami loyal et fraternel et homme du peuple. 
L'administration postcoloniale au Sénégal, en guise de trouver une solution à la sècheresse et famine qui ravagent le pays, accepte les dons caritatifs venant de l'Occident. Le griot écrivain contemporain africain se sert du griot personnage littéraire, Guelwaar pour condamner ouvertement et farouchement cette acceptation des dons vivriers. Il prononce un discours provocant lors d'un grand meeting où se trouvent les chefs et les notables des villages de la région ainsi que les représentants des organisations caritatives et des ambassadeurs. Selon Onyemelukwe : « Le déluge verbal de Guelwaar... tient à mettre en relief la prise de position d'Angèle, ce précurseur et progressiste dans le roman » (« Langage féministe » 260). Il démarre en affirmant :

L'index pointé à l'horizon, c'est pour indiquer le chemin à un étranger. Les cinq droits réunis, paume ouverte à un étranger, c'est mendier. C'est ce que nous faisons. Nos dirigeants nous ont rassemblés pour recevoir ces dons (Ousmane 139-140).

En un mot, il accuse les dirigeants du Sénégal à l'ère postindépendances d'avoir poussé le peuple sénégalais postcolonial à l'état de mendicité et de honte par la distribution des dons caritatifs. Son allocution provocatrice expose au peuple le symbolisme de ladite acceptation des dons alimentaires que voici :

- La mendicité aussi bien nationale qu'internationale. « Ce peuple de mendicité » (140).

- L'impuissance de l'Afrique à affronter ses innombrables problèmes sociaux, économiques et politiques.

- L'agenouillement, l'asservissement, l'assujettissement à perpétuité (de génération en génération), une nouvelle forme d'esclavage pour un peuple dit indépendant. 
- Le manque d'honneur et de dignité de l'homme noir. «Ces fréquentes distributions nous assassinent et tuent en nous toute velléité de dignité (140).

- L'économie de dépendance perpétuelle du Sénégal et de tout autre pays africain ayant une expérience semblable.

- La paresse du peuple africain.

- Le manque d'initiative et de capacité à diagnostiquer les problèmes socio-économiques qui entravent le pays et à proposer des solutions concrètes et honorables. Frantz Fanon a raison de dire qu'une véritable bourgeoisie n'existe pas dans les pays sous-développés comme le Sénégal et le Nigeria. Ces bourgeois manquent de capacité de grandes idées, d'inventivités. Il ajoute que la bourgeoisie nationale tourne de plus en plus le dos à l'intérieur, aux réalités du pays en friche et regarde vers l'ancienne métropole (108).

Aux dires de Guelwaar:

La sècheresse, la famine, les maladies ne sont pas des opprobres. L'opprobre, c'est lorsque ceux qui nous gouvernent ne sont pas capables de prévoir l'affrontement de la nature. L'opprobre c'est encore - comme nous, ici présents, lorsque tout un peuple attend qu'un autre peuple le nourrisse, l'habille, le soigne. Et ce peuple de mendiants, comme nos dirigeants, n'auront qu'un seul mot à répéterde génération en génération : «Jerejef ! Jerejef ! Merci ! Merci !» (140)

Sembène Ousmane, à travers la bouche du griot personnage littéraire, Guelwaar accuse ouvertement des autorités postcoloniales d'être responsables de la chute vertigineuse du continent africain 
dans le précipice de la faim, de la misère, et de la mendicité nationale voire internationale. L'un et l'autre jouent donc le rôle de critique des maux socioéconomiques et politiques de la société postcoloniale.

L'allocution de Guelwaar embellie de belles images : les comparaisons et d'autres formes de figures de rhétorique, démontre fortement qu'il s'agit d'un maître de la parole. Tout comme Ousmane, il maîtrise parfaitement la parole. Par l'entremise du griot personnage littéraire, Sembène Ousmane se penche à montrer à son peuple, en particulier et aux Africains, en général, ce qu'il y a de la honte, du manque de dignité et d'honneur dans l'acceptation des dons vivriers. Il veut éveiller aux Africains une prise de conscience de sa dignité et les exhorter à ne pas rester à la traîne des pays industrialisés, ce qui remonte à l'acceptation des dons alimentaires ; mais plutôt à penser au développement basé sur le travail collectif du peuple africain. Les deux types de griots dans Guelwaar se chargent donc de fonctions de porte-parole du peuple, d'éducateur du peuple et de libérateur du peuple.

Il est à remarquer que le discours agressif prononcé par Guelwaar finit par provoquer l'agression de Guelwaar par les agents de police menant inéluctablement à sa mort suite à une hémorragie interne. Le préfet Amadou Fall ordonne à ses gorilles de le faire taire pour toujours. Non seulement l'a-t-on tué, son corps a aussi disparu de la morgue par une erreur administrative. On enterre le feu griot, Guelwaar, un chrétien, dans un cimetière musulman du village des «(Ciss) ». Ce quiproquo risque de déboucher sur une guerre de religion entre deux communautés.

Le langage féministe de Guelwaar menant à sa victimisation, provoque une révolte collective : les jeunes hommes, les jeunes filles, les femmes et représentants des hommes religieux sont tous impliqués dans cette révolte qui consiste à vider les sacs, sachets ... 
importés des Etats-Unis ; de France, d'Italie, d'Allemagne, du Japon, de Hollande, et de Belgique (162). C'est un refus collectif des dons caritatifs. Voilà donc que le griot personnage littéraire qui finit par devenir un héros tragique, partage avec son créateur le griot écrivain contemporain Sembène Ousmane les fonctions de harangueur du discours violent et ennuyeux contre les autorités postcoloniales, de psychologue, d'incitant d'une révolte collective du peuple et de déconstructeur des préceptes religieux.

Scrutons cette scène de la grande réunion pour la distribution des dons vivriers. D'après le griot-narrateur, porte-parole de l'écrivain :

Guignane comme ceux de sa classe d'âge appelés « troisième âge » aujourd'hui ... s'alignaient face à une tribune décorée de drapeaux européens, en présence des ministres, des députés, des ambassadeurs, des chefs de village pour se voir distribuer un lot. Les vieux hommes se décoiffaient cheveux au soleil pour dire «Jerejef ! Jerejef ! Merci ! Merci ! (59)

Ces vieux Africains dits indépendants non seulement s'alignent-ils face à une tribune décorée de drapeaux européens ..., ils se décoiffent cheveux au soleil pour dire, Jerejef ! Jerejef ! Merci ! Merci ! Ousmane se montre satiriste de premier rang en peignant cette "scène d'humiliation, de dégradation, de honte, de l'être humain, d'une ethnie, brève, d'une race, la race noire » (Onyemelukwe, « Langage féministe » 357).

Notons que « les drapeaux européens » reflètent la présence - absence des Européens, des anciens occupants. Ils représentent les Blancs qui sont déjà partis, leurs sièges occupés par les Noirs africains mais dont l'influence se sent toujours en Afrique postcoloniale. Ceci avec la collaboration des dirigeants en Afrique postcoloniale, tels des ministres, des ambassadeurs, des chefs de 
village ici présents. Ousmane nous met en contact avec une excellente peinture satirique d'un peuple mendiant, qui tendent leurs battù pour obtenir des denrées vivrières : " cinq kilos de Rice UncleBen's, trois kilos de lait en poudre, de la farine, du sucre ... » (59).

Un intertexte apte qui vient à l'esprit ici c'est la scène de décoration du vieux Nègre Meka dans Le vieux nègre et la médaille de Ferdinand Oyono. Ce qu'il y a de différence c'est que cette dernière est pontifiée par la présence physique des Blancs - les maîtres coloniaux alors que la scène de distribution des dons caritatifs est marquée par la présence - absence des Blancs, ce que semble désigner l'image d'une tribune décorée de drapeaux européens. Ceci est le symbole de postcolonialisme ou le processus systématique de domination politique et culturelle occasionnée par l'imposition des structures de pouvoir impérialiste.

L'engagement féministe du griot écrivain contemporain africain dans Guelwaar ne s'arrête pas avec le support moral et la protection environnementale et légale qu'il accorde aux femmes de l'Association féminine - les Morom-yi, mais peut se déceler aussi à travers le fait qu'il fait le portrait de la femme africaine non seulement dans son rôle traditionnel d'épouse, de mère, de ménagère ou de servante, mais aussi dans son rôle de contributrice au progrès de la société où elle se trouve; en d'autres termes, la femme africaine en évolution, la femme africaine en lutte pour améliorer la condition féminine. Telles sont Dame Véronique et Angèle, première épouse de Guignane.

Angèle est aussi porte-parole du griot écrivain contemporain africain. C'est elle la première à condamner l'acceptation des dons vivriers ce qu'elle qualifie d'aumône (59). Elle fustige les patriarches dont son mari Guignane à travers un langage féministe tout en disant que : «L'indépendance est valable pour le père de 
famille qui nourrit sa famille.... S'il ne le peut pas, il n'est pas indépendant. Des épaves! Aucune dignité ! (60).

Ousmane dénonce la polygamie à travers la bouche d'Angèle. On peut sentir la voix de l'auteur de Guelwaar derrière Oumy, la plus jeune des veuves de Meysaa Ciss lorsqu'elle condamne le mariage forcé et l'héritage d'épouse. Elle est victime de ces deux formes de violence contre les femmes. Son père l'a forcée d'épouser Meysaa Cisssous forme de paiement d'une dette. A la mort dudit mari, son frère cadet veut l'hériter comme épouse. C'est à ce moment-là qu'elle se révolte contre ces pratiques nuisibles à la femme en s'enfuyant. Le griot écrivain contemporain africain dénonce toutes les pratiques socioculturelles ou religieuses qui entravent l'épanouissement et le progrès de la femme en société ; tout ce qui tient à humilier et à dégrader la femme. On peut décortiquer sa dénonciation de la soumission des épouses et filles sénégalaises à l'humiliation, ces femmes et filles obligées de danser pour plaire à l'audience lors de la distribution des dons alimentaires. C'est par la bouche de sa propre femme qu'il dénonce la prostitution. Même sa fille et son amie Helen mènent une vie de prostituée à Dakar. Sa femme lance une critique amère à cette pratique odieuse et brûle tous les pagnes que sa fille prostituée lui a achetés. Le griot personnage littéraire et le griot écrivain contemporain africain fonctionnent tous deux comme diseurs de la vérité, conseillers, et défenseurs de l'idéologie marxiste.

De surcroît, Sembène Ousmane en tant qu'écrivain de renommée mondiale qui a beaucoup écrit et en tant que réalisateur des films qu'il considère comme l'école du soir, sa meilleure manière d'éduquer son peuple dont la majorité est analphabète, il se charge incontestablement des fonctions d'historien, de bibliothèque publique bien documentée et de détenteur de la littérature écrite africaine postcoloniale. Sembène Ousmane en tant qu'écrivain 
contemporain africain se soucie du maintien de la sincérité dans ses affaires comme le vrai griot. Nous nous mettons d'accord avec Onyemelukwe que l'écrivain contemporain africain et le vrai griot aiment dire la vérité dans leurs œuvres que ce soit orale ou écrite. «Tous deux sont conscients du fait que leurs publics leur font confiance et donc afin de satisfaire ce but, ils s'efforcent d'être sincères, d'être sûrs dans toutes leurs activités. Voilà en quoi consiste 1'authenticité de leurs œuvres »(La littérature orale 73).

\section{Portraits du griot dans Guelwaar}

A partir des dix-huit fonctions du griot selon la peinture de Sembène Ousmane dans Guelwaar, notre étude aboutit aux dix-huit portraits du griot comme illustré dans le Tableau II ci-dessous. Ce sont : le griot porte-parole du peuple, le griot éducateur du peuple, le griot libérateur du peuple, le griot critique des maux socioéconomiques et politiques de la société, le griot ami loyal et fraternel et homme du peuple, le griot harangueur du discours violent et ennuyeux contre les autorités postcoloniales, le griot incitant de la révolte collective du peuple, le griot défenseur de l'idéologie marxiste, le griot promoteur de l'émancipation féministe, le griot déconstructeur des préceptes religieux, le griot maître de la parole, le griot diseur de la vérité, le griot historien, le griot psychologue, le griot conseiller, le griot éveilleur de la conscience sociopolitique chez les gens, le griot bibliothèque publique bien documentée, et le griot détenteur de la littérature écrite africaine postcoloniale. 
Tableau II : Portraits du griot dans Guelwaar de Sembène Ousmane

\begin{tabular}{|l|l|}
\hline $\mathbf{N}^{\mathbf{1}}$ & Portrait du griot \\
\hline 1. & Le griot porte-parole du peuple \\
\hline 2. & Le griot éducateur du peuple \\
\hline 3. & Le griot libérateur du peuple \\
\hline 4. & $\begin{array}{l}\text { Le griot critique des maux socioéconomiques et politiques } \\
\text { de la société }\end{array}$ \\
\hline 5. & Le griot ami loyal et fraternel et homme du peuple \\
\hline 6. & $\begin{array}{l}\text { Le griot harangueur du discours violent et ennuyeux } \\
\text { contre les autorités postcoloniales }\end{array}$ \\
\hline 7. & Le griot incitant de la révolte collective du peuple \\
\hline 8. & Le griot défenseur de l'idéologie marxiste \\
\hline 9. & Le griot promoteur de l'émancipation féminine \\
\hline 10. & Le griot déconstructeur des préceptes religieux \\
\hline 11. & Le griot maître de la parole \\
\hline 12. & Le griot diseur de la vérité \\
\hline 13. & Le griot historien \\
\hline 14. & Le griot psychologue \\
\hline 15. & Le griot conseiller \\
\hline 16. & $\begin{array}{l}\text { Le griot éveilleur de la conscience sociopolitique chez les } \\
\text { gens }\end{array}$ \\
\hline 17. & Le griot bibliothèque publique bien documentée \\
\hline 18. & $\begin{array}{l}\text { Le griot détenteur de la littérature écrite africaine } \\
\text { postcoloniale }\end{array}$ \\
\hline
\end{tabular}

\section{Conclusion}

La littérature postcoloniale africaine telle que le témoigne Guelwaar de Sembène Ousmane nous offre dix-huit fonctions du griot à partir desquelles émergent dix-huit portraits du griot dont le griot 
bibliothèque publique bien documentée, et le griot détenteur de la littérature écrite africaine postcoloniale. Ousmane y fait figurer deux types de griot à en juger par l'image du vrai griot et le postulat d'Onyemelukwe identifiant sept catégories de nouveaux griots. L'étude découvre seulement deux parmi ces sept catégories de griot, à savoir : le griot personnage littéraire nommé Guelwaar et le griot écrivain africain contemporain, l'auteur lui-même, Sembène Ousmane. Le métier du griot est confronté aux certains changements d'où l'effritement de personnage du griot d'antan. En dépit de cela, certaines ressemblances existent entre ces nouveaux griots et le vrai griot malgré leurs points de divergence.

Le griot écrivain contemporain africain ressemble beaucoup plus au griot d'antan surtout par le grand nombre de rôle qu'il joue en tant qu'écrivain. Concluons en disant que le griot écrivain contemporain africain parait être une meilleure version du griot d'antan par son raffinement occasionné par son contact avec l'école occidentale d'où son portrait de bibliothèque publique bien documentée contrairement au portrait du griot d'antan de bibliothèque publique non documentée. C'est une vérité incontournable qu'un vrai griot puisqu'analphabète lorsqu'il meurt, il est inhumé avec son savoir mais le griot écrivain contemporain africain puisqu'alphabétisé est bien documenté dans les bibliothèques etc., mettant ainsi son savoir à la portée du peuple de génération en génération. 
Ifeoma Mabel Onyemelukwe est professeur titulaire et spécialiste en français et en la littérature africaine d'expression française au Département de français à l'Université Ahmadu Bello, Zaria au Nigeria.

Abubakar Dauda Adamu est maîtrisant dans le Département de Français à Ahmadu Bello University, Zaria au Nigeria.

Chukwunonso Muotoo est Maître Assistant Niveau I au Département de langues modernes, à l'Université NnamdiAzikiwe, Awka au Nigeria 


\section{Euvres citées}

Ashcroft, Bill, Gareth Griffiths \& Helen Tiffin. Key Concepts in Post-colonial Studies. London: Routledge, 1998

Ashcroft, Bill, Gareth, Griffiths \& Helen Tiffin. The Empire Writes

Back: Theory and Practice in Postcolonial Literatures.

London: Routledge, 1989.

Diop, Majhémout. Histoire des classes sociales dans l'Afrique de l'ouest II : Le Sénégal. Paris : François Maspero, 1972.

Dumont, René. L'Afrique noire est mal partie. Paris : Seuil, 1962.

Fanon, Frantz. Les damnés de la terre. Paris : François Maspero, 1968.

Kouyaté, Mamadou. La variabilité dans quatre versions de l'épopée mandingue de Djibril TamsirNiane. Thèse de Doctorat, Bordeaux III, 2015.

Mishra, V et B. Hodge. «What is Post-colonialism? » P. Williams

et I. Chrisman, eds. Colonial Discourse and Post-colonial Theory. Cambridge: CUP, 1994.

Onyemelukwe, Ifeoma Mabel. Heroism and Antiheroismin Literature in French: Can you See? An inaugural lecture Zaria: Ahmadu Bello University, Zaria, 2015.

- - . "Migritude: Nouvelle direction de la littérature africaine. Ifeoma Mabel Onyemelukwe, ed. New Perspectives in African Literature and Criticism. Zaria: Department of French, Ahmadu Bello University, Zaria, 2015: 148-165.

- - -. Violence \& Politics in Postcolonial Literature. Zaria: Labelle Educational Publishers, 2009.

- - - « Langage féministe et effet paradoxal dans Guelwaar de Sembène Ousmane. » NEOHELICON 30.1 (2003) : 257-262. Onyemelukwe, Ifeoma et Patience AjeibiOdeh. « La migritude : Un humanisme du XXIe Siècle. » NJOFLALS: Nsukka Journal of 
Foreign Languages and Literary Studies Maiden Edition (2018) : 1-20

Oxford English Dictionary. Oxford: OUP, 1989.

Ousmane,Sembène. Guelwaar. Paris: PA, 1996. 